\title{
On a variant of Newton-Coulomb's law
}

\author{
Alain Haraux \\ Sorbonne Université, Université Paris-Diderot SPC, CNRS, INRIA, \\ Laboratoire Jacques-Louis Lions, LJLL, F-75005, Paris, France. \\ e-mail: haraux@ann.jussieu.fr
}

December 30, 2020 


\begin{abstract}
A variant of the usual formulas for gravitational and electrostatic fields, differing from those by a logarithmic term, is introduced in order to solve some questions connected with a possible atomic contraction phenomenon at large time scale and the so-called hidden mass problem in cosmology. This approach is conceptually related to the MOND theory of M. Milgrom but allows a reversal of attracting forces when the distance becomes very small. The asymptotic behavior of solutions of a related dissipative ODE is studied, we obtain that all bounded trajectories converge to a point where the field vanishes.
\end{abstract}

Key words: attracting field, logarithmic perturbation, reversal of force, dissipative ODE. 


\section{Introduction}

The usual equation for both motions of a single planet around the sun and electrons in the deterministic Rutherford-Bohr atomic model is conservative with a singular potential at the origin. The basic equation

$$
m u^{\prime \prime}=-\frac{q^{2}}{4 \pi \varepsilon_{0}} \frac{u}{|u|^{3}}
$$

models Coulomb's central force (with $q$ the elementary charge, $\mathrm{m}$ the mass of the electron and $\varepsilon_{0}$ the vacuum permittivity) while for planets Newton's law has the form

$$
u^{\prime \prime}=-G M_{S} \frac{u}{|u|^{3}}
$$

where $G$ is the gravitational constant and $M_{S}$ the mass of the sun. Both equations are written in complex form in the orbital plane with a suitable set of axes and length unit, and are special cases of the conservative problem

$$
u^{\prime \prime}+c_{0} \frac{u}{|u|^{3}}=0 .
$$

In [8] the author considered the modified ODE

$$
u^{\prime \prime}+\delta u^{\prime}+c_{0} \frac{u}{|u|^{3}}=0,
$$

a model devised as an attempt to understand the possible long term effect effect of shocks with wandering particles on the behaviour of atoms and to explain strange phenomena recalled in [8] (cf. also $[1,12,5,11,15,17,18,16,19]$ for very important related considerations). Under the smallness assumption

$$
\left|u_{0}\right|\left|u_{0}^{\prime}\right|^{2}<2 c_{0} .
$$

on the initial data, it was shown that the trajectory of the solution is bounded and

$$
\lim _{t \rightarrow+\infty}|u(t)|=0 .
$$

In other terms the electron falls on the nucleus, leading to a kind of minimal "black hole". The status of matter inside black holes is nowadays still unknown, and some physicists even consider that if the singularity makes sense mathematically, when the particles collapse together the nature of matter is altered in such a way that its behaviour falls outside the application of the usual laws of Physics. Another alternative would be to imagine that the collapse cannot really happen and particles have to rebound when they are too close from the singularity. This might be consistent with some recent findings in star collapse such as [13].

In the present paper, we build a simple variant of the Newton-Coulomb potential which has two properties: near the singularity the attractive force becomes repulsive, and for large distances the attraction is slighter stronger than the force given by Newton-Coulomb's law. This will lead us to consider the slightly more complicated equation

$$
u^{\prime \prime}+\delta u^{\prime}+\gamma \frac{\ln (\alpha|u|) u}{|u|^{3}}=0,
$$

where $\gamma>0, \delta \geq 0$ and $\alpha>0$ is homogeneous to the inverse of a length. 
The plan of the paper is as follows: in Section 2 we briefly describe the circular orbits of the conservative version of $(1.5)$ (when $\delta=0$ ). In Section 3 , general results for the solutions of equation (1.5) are derived by very simple methods, this being made possible by the fact that no solution approches the singularity. Section 4 is devoted to the connection between this new equation and the physical question which motivated its introduction.

\section{The conservative case.}

Let us first consider the simple case of equation (1.1). Although in the case of solar system, the interesting trajectories are ellipses, more or less close to a circle depending on the planet, in the Rutherford model (cf.[17]), which served as a basis for Bohr's model [1] of the hydrogen atom, circular trajectories were privileged. The solutions travelling on a circular orbit

$$
|u(t)|=R
$$

have the form

$$
u(t)=R \exp \left(i \omega\left(t+t_{0}\right)\right.
$$

with

$$
\omega^{2}=\frac{c_{0}}{R^{3}} .
$$

In particular the constant velocity of the electron is

$$
\left|u^{\prime}(t)\right|=\omega R=c_{0}^{1 / 2} R^{-1 / 2} .
$$

This is consistent with the third Kepler's law for the planets motion driven by the gravitational field of the sun.

We now consider the conservative equation

$$
u^{\prime \prime}+\gamma \frac{\ln (\alpha|u|) u}{|u|^{3}}=0,
$$

which can, in the complex plane, be written as

$$
u^{\prime \prime}+\nabla V(u)=0,
$$

with

$$
V(u)=-\gamma \frac{\ln (e \alpha|u|)}{|u|} .
$$

In this case we find circular trajectories of radius $R$ only when $R>\frac{1}{\alpha}$, of the form (2.2) where now

$$
\omega^{2}=\frac{\gamma \ln \alpha R}{R^{3}} .
$$

In particular the constant velocity of the solution is now

$$
\left|u^{\prime}(t)\right|=\omega R=\gamma^{1 / 2}[\ln (\alpha R)]^{1 / 2} R^{-1 / 2} .
$$

It would be of interest to study the shape of non-circular bounded orbits and to determine, in particular, whether they are still periodic as in the case of equation (1.1).

In the next section we shall summarize the general properties of solutions for both equations (2.4) and (1.5). Note that (2.4) is (1.5) with $\delta=0$. 


\section{General results for equation (1.5)}

In this section, we investigate the standard relevant questions for the solutions of (1.5): wellposedness, boundedness and asymptotics.

\subsection{A global existence result}

We now state our first result

Theorem 3.1. For any $\left(u_{0}, u_{0}^{\prime}\right) \in \mathbb{C}^{2}$ with $u_{0} \neq 0,(1.5)$ has a unique global solution $u \in$ $C^{2}\left(\mathbb{R}^{+} \mathbb{C}\right)$ such $u(0)=u_{0}$ and $u^{\prime}(0)=u_{0}^{\prime}$. In addition $u^{\prime}$ is bounded and $u$ is bounded away from 0.

Proof. The existence and uniqueness of local non-vanishing solutions is obvious. Let us introduce the energy function $E$, defined as long as the solution $u$ exists and does not vanish by the formula:

$$
E(t):=\frac{1}{2}\left|u^{\prime}\right|^{2}(t)-\gamma \frac{\ln (e \alpha|u(t)|)}{|u(t)|} .
$$

We have immediately

$$
E^{\prime}(t)=-\delta\left|u^{\prime}(t)\right|^{2}
$$

In particular for all $t \in[0, \operatorname{Tmax})$ we have

$$
\frac{1}{2}\left|u^{\prime}\right|^{2}(t)-\gamma \frac{\ln (e \alpha|u(t)|)}{|u(t)|} \leq E_{0}:=E(0)
$$

which implies in particular the two inequalities

$$
\forall t \in\left[0, T_{\max }\right), \quad\left|u^{\prime}\right|^{2}(t) \leq 2\left(E_{0}+\gamma \sup _{s>0} \frac{\ln (e \alpha s)}{s}\right)<\infty
$$

and

$$
\forall t \in\left[0, T_{\max }\right), \quad \gamma \frac{\ln (e \alpha|u(t)|)}{|u(t)|} \geq-E_{0} .
$$

The conclusions follow immediately

\subsection{Bounded trajectories}

It is reasonable to imagine that both equations (2.4) and (1.5) have some unbounded solutions. On the other hand we have the following immediate result.

Theorem 3.2. Let $u_{0} \neq 0$ and assume the condition

$$
\left|u_{0}\right|\left|u_{0}^{\prime}\right|^{2}<2 \gamma \ln \left(e \alpha\left|u_{0}\right|\right) .
$$

Then the global solution $u$ of (1.5) with initial conditions $u(0)=u_{0} ; u^{\prime}(0)=u_{0}^{\prime}$ is bounded.

Proof. This condition is equivalent to $E_{0}<0$. Then by (3.3) we find that $u(t)$ is a priori bounded since

$$
\lim _{s \rightarrow \infty} \frac{\ln (e \alpha s)}{s}=0 .
$$

Remark 3.3. Condition (3.4) can be viewed as a smallness condition on $u_{0}^{\prime}$ for $\left|u_{0}\right|$ fixed. If $\gamma$ is large the restriction becomes less stringent. 


\subsection{Asymptotics of the bounded solutions}

Theorem 3.4. Assume $\delta>0$ Then for any bounded solution $u$ of (1.5) on $\mathbb{R}^{+}$, we have

$$
\lim _{t \rightarrow+\infty}|u(t)|=\frac{1}{\alpha} .
$$

More precisely the exists $\zeta \in \mathbb{C}$ such that

$$
|\zeta|=\frac{1}{\alpha} \quad \text { and } \quad \lim _{t \rightarrow+\infty} u(t)=\zeta .
$$

Proof. Equation (1.5) can be written as

$$
u^{\prime \prime}+\nabla V(u)+\delta u^{\prime}=0
$$

where the potential $V$ is analytic in a neighborhood of the closure of the trajectory. The result then follows from Theorem 1.1 of [9], cf. also [10].

\section{Some limited potential applications to physics.}

1) The initial idea for considering the potential $V(u)=\gamma \frac{\ln (e \alpha|u|)}{|u|}$ and its associated central acceleration field

$$
\vec{G}=-\gamma \frac{\ln (\alpha|u|) u}{|u|^{3}}
$$

was to encompass at the same time a MOND-like model (cf. [14]) and the idea that the gravitation field may become repulsive at very short distances, of the order of the size of atoms. Indeed, compared to Newton's formula, the logarithmic term introduces almost invisible variations when one considers distances comprised between the size of a planet and a few light years, when the characteristic length $r=\frac{1}{\alpha}$ is of the order of an atom's radius. In the previous formula the mass $M$ of the central object is included, and we can assume $\gamma=C M$ where $C$ has the dimensionaltity of a gravitational constant, so that the formula should be understood as

$$
\vec{G}=-C M \frac{\ln (\alpha|u|) u}{|u|^{3}}
$$

In this model the gravitation force vanishes when $|u|=\frac{1}{\alpha}:=r_{0}$ and we may assume, for simplicity that the supposedly universal gravitational constant $\Gamma$ is obtained when $|u|=1 A U$. Let us see what happens when we take for $r_{0}$ the value $10^{-10} \mathrm{~m}=1 \AA$. This means $\alpha=10^{10} \mathrm{~m}^{-1}$ and therefore $C \ln \left(10^{10} \cdot 1,510^{11}\right)=\Gamma$ or

$$
\Gamma \sim 48.76 C
$$

At a distance of 10 millions of light years (the radius of the Coma cluster), the fictitious gravitational constant generated by our formula will be approximately

$$
\Gamma^{*}=C \ln \left(10^{10} \cdot 10^{23}=33 C \ln 10\right) \sim 76 C
$$

so that

$$
\frac{\Gamma^{*}}{\Gamma} \sim 1.6
$$


This is not sufficient to explain the remaining factor 5 remaining in the "missing mass enigma" after reduction of the extraneous sources of discrepancy studied in [6], but it is not completely negligible. At the level of galaxies (to explain the flat rotation curves), the amplification factor will be reduced by an extra factor $31 / 33$ and will become 1.5. It may help but it is not sufficient in itself.

2) Concerning the atomic contraction hypothesis, it was suggested in [7] and [8] that a reversal of the electro-static forces at short distances may help to understand why the atoms do not collapse completely. In this case we may imagine that $\frac{1}{\alpha}=r_{0}$ has the order of magnitude of the diameter of a proton, about $10^{-15} \mathrm{~m}$ or more probably something between $10^{-15} \mathrm{~m}$ and $10^{-10} \mathrm{~m}=1 \AA$. The interesting point here is that Theorem 3.4 indicates a contraction property of atoms towards a radius $r_{0}$, at an unknown rate but with convergence of the electron to a motionless state, which is a little bit counter-intuitive.

Remark 4.1. Of course we are unable to exhibit any mechanism that would generate a force field of the form studied in this text. For the classical Newton gravitational law, some potential explanations have been proposed even at the time of Newton himself (cf. [2, 3, 4, 6]) but for the time being no mechanism is recognized by the scientific community as being the certain cause of the gravitational field. As long as the cause is unknown, speculations about the exact form of the law are still admissible.

\section{Conclusion.}

In this short note, we studied in a sense the simplest possible model involving a logarithmic divergence from the classical Newton-Coulomb force field. Other models are of course possible, which would produce a stronger divergence of the fictitious gravitational constant, for instance

$$
\vec{G}=-C M \frac{\ln ^{3}(\alpha|u|) u}{|u|^{3}}
$$

But it is probably not so urgent to investigate such more complicated models as long as the attraction reversal is not proven by experiments, which seems to be technically difficult for both gravitational and electro-static force fields.

\section{References}

[1] N.Bohr, On the Constitution of Atoms and Molecules, Philosophical Magazine, Series 26, vol. 1, (July 1913) , p.1-24.

[2] Н.Снавот; Georges-Louis Lesage (1724-1803): a theoretician of gravitation in search of legitimacy, Arch. Internat. Hist. Sci. 53 (2003), no. 150-151, 157-183.

[3] M.R. Edwards; Pushing gravity: New perspectives on Lesage's theory of gravitation Apeiron, 2002, In: Revue d'histoire des sciences, tome 58, n2, 2005. pp. 519-520.

[4] M.R. EdWARDs; Photon-graviton recycling as cause of gravitation Apeiron.14, 3 (2007), $214-230$. 
[5] G. GaliLei; Two new sciences, A new translation with introduction and notes by Stillman Drake, The University of Wisconsin Press (1974).

[6] A.Haraux, About Dark Matter and Gravitation. Preprints 2020, 2020070198 (doi: 10.20944/preprints202007.0198.v1).

[7] A.Haraux, On Carboniferous Gigantism and Atomic Shrinking. Preprints 2020, 2020110544 (doi: 10.20944/preprints 202011.0544.v2).

[8] A.Haraux, On some damped 2 body problems, Preprints 2020, 2020120510 (doi: 10.20944/preprints202012.0510.v2). To appear in Evolution Equations and Control Theory.

[9] A. Haraux, M.A. Jendoubi, Convergence of solutions of second-order gradient-like systems with analytic nonlinearities. J. Differential Equations 144 (1998), no. 2, 313-320.

[10] A. Haraux, M.A. Jendoubi, The convergence problem for dissipative autonomous systems. Classical methods and recent advances. Springer Briefs in Mathematics, Springer, Cham; BCAM Basque Center for Applied Mathematics, Bilbao, 2015. xii+142 pp. ISBN: 978-3-319-23406-9; 978-3-319-23407-6

[11] J.F.Harrison, A.Kaiser and J.M. VAndenBrooks; Atmospheric oxygen level and the evolution of insect body size. Proceedings of the Royal Society B. 277(2010):1937-1946. doi:10.1098/rspb.2010.0001

[12] E.Hubble, M.L. Humason, The Velocity-Distance Relation among Extra-Galactic Nebulae, Astrophysical Journal, vol. 74, p. 43.

[13] L.Mersini-Houghton, Backreaction of Hawking Radiation on a Gravitationally Collapsing Star I: Black Holes? Physics Letters B 738(C) (2014), 61-75.

[14] M. Milgrom, A modification of the Newtonian dynamics as a possible alternative to the hidden mass hypothesis, The Astrophysical Journal, 270 (1983 ):365-370.

[15] Ryssa Parks; An Overview of Hypotheses and Supporting Evidence Regarding Drivers of Insect Gigantism in the Permo-Carboniferous, Western Washington University reports, Spring 2020.

[16] R. Penrose ; The Big Bang and its Dark-Matter Content: Whence, Whither, and Wherefore, Found Phys (2018) 48:1177-1190.

[17] E. Rutherford; The Scattering of $\alpha$ and $\beta$ Particles by Matter and the Structure of the Atom, E. Rutherford, F.R.S. Philosophical Magazine, Series 6, vol. 21 May 1911, p. $669-688$

[18] E. Schrodinger; An Undulatory Theory of the Mechanics of Atoms and Molecules, Phys. Rev. vol. 28, no 6, decembre 1926, p. 1049-1070 (DOI 10.1103/PhysRev.28.1049)

[19] F. Zwicky; The redshift of extragalactic nebulae, Helvetica Physica Acta, Vol. 6 (1933), $110-127$. 